\title{
Transurethral Resection of Bladder Tumor
}

National Cancer Institute

\section{Source}

National Cancer Institute. Transurethral Resection of Bladder Tumor. NCI Thesaurus.

Code C116651.

A surgical procedure used to treat bladder tumors, during which a resectoscope is passed through the urethra and into the bladder. 